\title{
Optimization of Single Pulse Control based on Design of Experiments for Switched Reluctance Machines
}

\author{
Duc Pham ${ }^{* a)}$ \\ Georg Tobias Götz ${ }^{*}$ Non-member, \\ Leo Vijay Pugazhenthi** \\ Non-member \\ Rik W. De Doncker* \\ Non-member
}

(Manuscript received Feb. 1, 2021, revised July 1, 2021)

J-STAGE Advance published date : Aug. 27, 2021

\begin{abstract}
Owing to the robust structure of switched reluctance machines, high-speed operations are preferred to achieve higher power densities. Generally, at high speeds, single pulse control is applied. However, its control parameter set is not unique. This paper proposes an algorithm based on design of experiments that simultaneously allows the calculation of optimal control parameters for an operating area and the minimization of the total machine losses. The designed experiments are finite-element analyses (FEAs) considering both iron and copper losses. A Pareto search algorithm solves the proposed multi-objective optimization problem. The results for different response surfaces are compared considering the errors in torque production and machine loss prediction. A reduced response surface based on 15 FE-runs shows a mean error of $1.9 \%$, whereas the maximum error is $6.3 \%$ for machine losses within the validation operating area.
\end{abstract}

Keywords: design of experiment, single pulse control, switched reluctance machine, response surface

\section{Introduction}

Due to the simple geometrical structure, switched reluctance machines (SRMs) are mainly operated at high speeds allowing higher power densities ${ }^{(1)}$. With higher speeds the back-emf increases reducing the capability of an active phase current control. Instead, single pulse control (SPC) aims to magnetize and demagnetize each phase such that a reference average torque is provided. However, the current waveforms have a pulsating nature with high current slopes. This, in turn, does not only lead to higher iron losses but also to distinctive copper ac losses in the windings. The combinations of the control parameters are not unique for a considered operating point. Different sets of turn-on $\theta_{\text {on }}$, turn-off angles $\theta_{\text {off }}$ and freewheeling duration $\Delta \theta_{\mathrm{fw}}$ provide the same torque at the same speed. Nevertheless, the current waveform differs and, thus, both the iron and copper losses are different. The considered control parameters are depicted in Fig. 1.

This paper presents a method based on design of experiments (DOE) to find the optimal control parameters for SRMs controlled by SPC with minimized iron and copper losses. This paper's objective is to statistically map the input parameters shown in Fig. 1 and the machine speed to outputs

This paper is based on ${ }^{(12)}$, which was published in the Proceedings of the International Conference on Electrical Machines and Systems (2020) 2020 (CIEEJ

a) Correspondence to: Duc Pham. E-mail: duchuu.pham@isea. rwth-aachen.de

* Institute for Power Electronics and Electrical Drives (ISEA), RWTH Aachen University

Jägerstrasse 17/19 - 52066 Aachen - Germany

** Valeo Siemens eAutomotive

Siemensstraße 15, 97616 Bad Neustadt a.d. Saale Germany

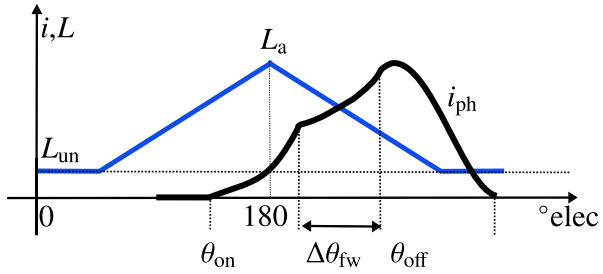

Fig. 1. Current waveform of SRG with SPC

of interests. The copper, iron and mechanical losses are used as output values in order to find the minimal losses. Especially, the eddy-current losses within the windings are speed dependent. Therefore, not only the control parameters of SPC are considered, but the machine speed $n$ is also considered as an independent input variable for the optimization problem. Furthermore, the provided torque is considered as an output variable in order to set a specific operating point.

\section{Optimal Single Pulse Control}

This paper concentrates on control optimization for switched reluctance generators (SRGs). In this work, an asymmetric half-bridges inverter is considered. Furthermore, only the generator mode is investigated. Thus, the SRM driven by the inverter is also referred as SRG within this work. Fig. 1 shows the phase current $i_{\mathrm{ph}}$ and the unaligned and aligned phase inductance $L_{\mathrm{un}}$ and $L_{\mathrm{a}}$. At the turn-on angle $\theta_{\mathrm{on}}$ a machine phase is magnetized by applying the positive dc-link voltage $V_{\mathrm{dc}}$ to one phase. During the freewheeling phase $\Delta \theta_{\mathrm{fw}}$ one of the switches in the asymmetric half-bridge is closed. Therefore, no voltage is applied to the phase. Eventually, applying the negative dc-link voltage $-V_{\mathrm{dc}}$ at the turn-off angle $\theta_{\text {off }}$ demagnetizes the phase allowing the 
phase current $i_{\text {ph }}$ decreases to $0 \mathrm{~A}$.

In (2), optimal control angles $\theta_{\text {on }}$ and $\theta_{\text {off }}$ are calculated to maximize machine efficiency. Furthermore, (3) investigated the efficiency improvements with the freewheeling period $\Delta \theta_{\mathrm{fw}}$. However, both investigations do not consider eddycurrents in the coils. Especially, for SRGs with high-speed operation high eddy-current losses occur depending on the current waveform, the electrical frequency and the coil geometry. In (1), an empirical model of the eddy-current losses based on finite element analysis (FEA) was derived to adjust the freewheeling angle for machine losses minimization. This paper proposes a DOE approach to cope with all control parameters at the same time. In addition, no physical equation for the machine losses is derived or fitted. Instead, a statistical model based on FEA experimental runs is used for optimization of the control parameters. Hence, both the iron losses and the dc and ac losses in the copper coils can be considered with the precision of FEAs.

\section{Methods}

3.1 Design of Experiments The objective of DOE is to generate a relation between in- and output parameters. The challenge of an efficient response surface (RS) design is the generation of a minimal experimental set, which is still able to accurately represent the in- and output behavior. In this work, central composite design (CCD) ${ }^{(4)}$ is applied, which is suitable to fit a full quadratic model and to run sequential experiments. CCD applies three different methods for generating sample points for an experimental run. Fig. 2 shows the sample points for two design variables. Firstly, each design variable has two levels $(+1$ and -1$)$ based on full factorial design. The factorial points (black dots) allow an estimation of first-order and interaction terms. In addition, for each design variable the median is considered. Eventually, additional points above and below the median values represent axial points, which allow an estimation of squared terms. For the circumscribed CCD type, the red stars depict axial points with sequential replacement of each design variable by $\pm \alpha$ from the center point. Hence, these points are outside the hypercube spanned by factorial design. Instead, the inscribed CCD type can be obtained by dividing all factor levels by $\alpha$. The total number of experiments with CCD is calculated by

$$
N_{\mathrm{CCD}}=2^{k}+2 k+c p
$$

with the number of design variables $k$, and the number of replicates of the center points $c p$. The total number of axial points is $2 k$. The number of factorial runs is calculated by $2^{k}$.

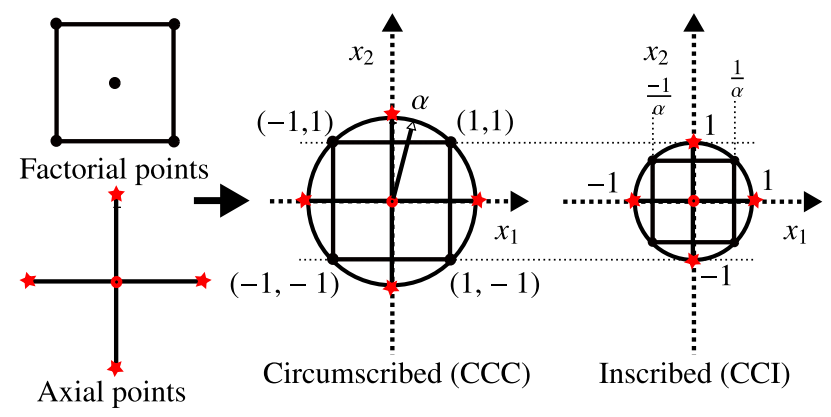

Fig. 2. Circumscribed and inscribed CCDs
In order to minimize the number of experiments, it is sufficient to consider one replicate, i.e. $c p=1$. The distance $\alpha$ is obtained by (4)

$$
\alpha=\left[2^{k}\right]^{\frac{1}{4}}
$$

For circumscribed CCD the axial points are outside the defined parameters limits. Thus, in the following inscribed $\mathrm{CCD}$ is considered, since it keeps the predefined parameter limits.

3.2 Loss Calculation Besides the objective to accurately set the machine to a defined operating point, determined by the reference machine speed $n^{*}$ and the torque $T^{*}$, the control parameter set also needs to minimize the machine's total losses. Generally, iron losses occur in the components of an electrical machine consisting of steel sheets. Not only do these losses depend on the injected current waveforms, but also on the machine's geometry and material. Hence, this paper utilizes FEAs for the calculation of iron losses, that consists of hysteresis and eddy-current losses

$$
P_{\mathrm{Fe}}=P_{\mathrm{Hys}}+P_{\mathrm{Eddy}}, \cdots \ldots \ldots \ldots \ldots \ldots \ldots \ldots \ldots \ldots \ldots \ldots
$$

where the hysteresis losses are calculated from the time series data of local magnetic flux densities $B_{\mathrm{k}}$ of the k-th loop for each direction in each finite element volume $V_{\mathrm{e}}{ }^{(5)}$

$$
P_{\mathrm{Hys}}=\sum_{n=1}^{n_{\text {elem }}}\left[f \cdot \sum_{m=1}^{n_{\text {loop }}} a\left(B_{\mathrm{k}}\right) \cdot V_{\mathrm{e}}\right] \ldots \ldots \ldots \ldots \ldots \ldots
$$

$n_{\text {elem }}$ is the number of elements, $n_{\text {loop }}$ the number of loops, $f$ the base frequency, and $a\left(B_{\mathrm{k}}\right)$ is obtained by frequency separation method ${ }^{(5)}$. The FEA considers the thickness of the steel sheets by performing a one-dimensional magnetic field analysis in the lamination direction for each volume element. With the electric resistivity of the steel sheets, the FEA calculates the eddy-current loss $P_{\text {eddy. }}$ In this paper, the material NO10 with a thickness of $0.1 \mathrm{~mm}$ is considered ${ }^{(6)}$.

In (7), SRMs show high copper losses in the coils near the air gap. The injected currents not only cause dc ohmic losses, but they also cause additional losses in the windings due to the skin- and proximity effect. Hence, the copper losses do not only depend on the geometrical form and placement of the windings, but they also depend on the injected current waveforms. In Fig. 3 the current displacement in the windings is depicted.

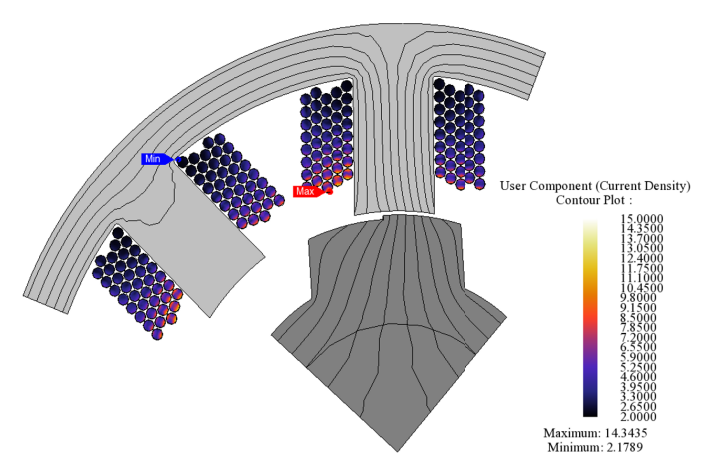

Fig. 3. A quarter of an 8/4-SRG with current density distribution in coils in $\mathrm{A} \mathrm{mm}^{-2}$ for nominal operating point 
Table 1. Parameter limits for DOE

\begin{tabular}{llll}
\hline \multirow{2}{*}{ Parameter } & \multicolumn{2}{c}{ Limits } & Unit \\
& Min & Max & \\
\hline$\theta_{\mathrm{on}}$ & 100 & 190 & $\mathrm{el}$ \\
$\theta_{\mathrm{off}}$ & 230 & 300 & $\mathrm{el}$ \\
$\Delta \theta_{\mathrm{fw}}$ & 10 & 40 & $\mathrm{el}$ \\
$n_{\mathrm{SRG}}$ & 20000 & 30000 & $\mathrm{rpm}$ \\
\hline
\end{tabular}

Eventually, electrical machines also generate mechanical losses due to friction. In (8), an empirical model estimates the windage losses for salient pole machines

$$
P_{\mathrm{Wdg}}=K_{\mathrm{sal}} \cdot \pi \cdot C_{\mathrm{d}} \cdot \rho_{\mathrm{air}} \cdot \omega_{\mathrm{r}}^{3} \cdot R_{\mathrm{rotor}}^{4} \cdot L_{\mathrm{stk}}, \cdots \cdots
$$

with the skin friction coefficient $C_{\mathrm{d}}$, the air density $\rho_{\text {air }}$, the rotational speed $\omega_{\mathrm{r}}$ in $\mathrm{rad} \mathrm{s}^{-1}$, the rotor radius $R_{\text {rotor }}$ and the stack length $L_{\text {stk }}$. In this paper, the SRM is assumed to have shrouds on both sides. Generally, rotor shrouds impede axial air flow and, thus, reduce the windage losses ${ }^{(8)}$. Hence, the saliency factor is set to $K_{\mathrm{sal}}=\frac{3}{2}$. Finally, the sum of all losses represents the total machine losses

$$
P_{\mathrm{Loss}}=P_{\mathrm{Cu}}+P_{\mathrm{Fe}}+P_{\mathrm{Wdg}},
$$

where the iron losses $P_{\mathrm{Fe}}$ and copper losses $P_{\mathrm{Cu}}$ are calculated with FEAs, while the windage losses $P_{\mathrm{Wdg}}$ are analytically calculated.

3.3 Generation of Response Surfaces The objective of the response surface (RS) is to generate an analytical representation between the control input parameters and defined output parameters. In total, three control parameters and the machine speed can be considered as independent variables with the limits in Table 1. Since the total objective is both an accurate torque generation and a minimization of the total losses of the SRM, the resulting torque $T_{\mathrm{m}, \mathrm{RS}}$ and machine losses $P_{\mathrm{RS}}$ are defined as outputs. Note that a larger interval for each design variable also leads to a wider range of possible torque and machine loss outputs. This may lead to non relevant or even unfeasible operating points, which again decreases the accuracy of the RS. Here, the nominal speed of $25 \mathrm{krpm}$ is also the center of the speed interval.

In this paper, a second-order RS given by

$$
f_{\mathrm{RS}}(\boldsymbol{x})=\beta_{0}+\sum_{i=1}^{N_{\mathrm{D}}} \sum_{j \geq i}^{N_{\mathrm{D}}} \beta_{\mathrm{i}, \mathrm{j}} \cdot x_{\mathrm{i}} x_{\mathrm{j}}+\epsilon \cdots \cdots \cdots \cdots \cdots
$$

approximates the relationship between the independent variables in Table 1 and the depended variables namely the torque $T_{\mathrm{m}, \mathrm{RS}}$, the copper losses $P_{\mathrm{Cu}, \mathrm{RS}}$, the iron losses $P_{\mathrm{Fe}, \mathrm{RS}}$ and the windage losses $P_{\mathrm{Wdg}, \mathrm{RS}} . N_{\mathrm{D}}$ indicates the number of design variables and $\epsilon$ the error term. In order to evaluate the error of the RS, two measures are considered. The root mean squared error (RMSE) and the $R^{2}$ are described by the evaluation of the RS $f_{\mathrm{i}, \mathrm{RS}}$ for the i-th experiment $f_{\mathrm{i}}$, the outputs from the corresponding FEA results $y_{\mathrm{i}}$ and the mean value $\bar{y}$

$$
\begin{aligned}
& \text { RMSE }=\sqrt{\frac{1}{N_{\mathrm{D}}} \sum_{i=1}^{N_{\mathrm{D}}}\left(y_{\mathrm{i}}-f_{\mathrm{i}, \mathrm{RS}}\right)^{2}} \ldots \ldots \ldots \ldots \ldots \ldots \ldots \ldots \ldots \ldots \ldots
\end{aligned}
$$

3.4 Multi-Objective Optimization The objective is not only to find optimal control parameters to minimize the machine losses, but also the error to a desired operating point. A reference operating point is defined by the machine speed $n^{*}$ and the machine average torque $T^{*}$. The optimization problem for the multiple-objective optimization (MOO) is defined as

$$
\begin{aligned}
& \min f_{\text {Loss }}\left(\boldsymbol{x}_{\mathrm{ctrl}}\right)+f_{\mathrm{OP}}\left(\boldsymbol{x}_{\mathrm{ctrl}}\right) \\
& \text { subject to } x_{\mathrm{i}, \mathrm{lb}} \leq x_{\mathrm{i}, \mathrm{ctrl}} \leq x_{\mathrm{i}, \mathrm{ub}} \text { for } i=1, \ldots, N_{\mathrm{D}}-1 \\
& \text {................ (11) } \\
& n_{\mathrm{SRG}}=n^{*} \ldots \ldots \ldots \ldots \ldots \ldots \ldots \ldots \ldots \ldots \ldots \ldots \ldots \ldots \ldots
\end{aligned}
$$

The lower and upper bounds $x_{\mathrm{i}, \mathrm{lb}}$ and $x_{\mathrm{i}, \mathrm{ub}}$, respectively, are the minimum and maximum values from Table 1 . Note that the machine speed $n_{\mathrm{SRG}}$ is set equal to the reference speed $n^{*}$. Consequently, this allows a fixation of one operating point value. The first objective function in the cost function represents the total machine losses with a weighting factor $w_{\text {Loss }}$ and a scaling factor $\hat{P}_{\text {Loss }}$ :

$$
f_{\text {Loss }}\left(\boldsymbol{x}_{\mathrm{ctrl}}\right)=w_{\text {Loss }} \cdot \frac{P_{\text {Loss }, \mathrm{RS}}\left(\boldsymbol{x}_{\mathrm{ctrl}}\right)}{\hat{P}_{\text {Loss }}} \ldots \ldots \ldots \ldots \ldots
$$

Note that $P_{\text {Loss,RS }}$ is composed of individual RSs for every loss component

$P_{\mathrm{Loss}, \mathrm{RS}}\left(\boldsymbol{x}_{\mathrm{ctrl}}\right)=P_{\mathrm{Cu}, \mathrm{RS}}\left(\boldsymbol{x}_{\mathrm{ctrl}}\right)+P_{\mathrm{Fe}, \mathrm{RS}}\left(\boldsymbol{x}_{\mathrm{ctrl}}\right)+P_{\mathrm{Wdg}, \mathrm{RS}}\left(\boldsymbol{x}_{\mathrm{ctrl}}\right)$.

The second objective function gives the absolute difference between the reference average torque and the average torque calculated by the RS

$$
f_{\mathrm{OP}}\left(\boldsymbol{x}_{\mathrm{ctrl}}\right)=w_{\mathrm{OP}} \cdot \frac{\left|T^{*}-T_{\mathrm{m}, \mathrm{RS}}\left(\boldsymbol{x}_{\mathrm{ctrl}}\right)\right|}{\hat{T}_{\mathrm{m}}}, \cdots \ldots \ldots \ldots
$$

with a weighting factor $w_{\mathrm{OP}}$ and a scaling factor $\hat{T}_{\mathrm{m}}$. This objective function aims to set the machine average torque equal to the desired torque $T^{*}$. The weighting factors are set to $w_{\mathrm{OP}}=w_{\text {Loss }}=1$ in order to equally minimize the total machine losses and the torque error.

3.5 Workflow The complete workflow is summarized in Fig. 4. The first step is to select the independent input variables of interests and to define their appropriate limits. Note that the selection of the limits impacts the quality of the RS. Next, the current waveforms of each SRG phase are generated with MATLAB Simulink ${ }^{(9)}$ and serve as inputs in JMAG ${ }^{(10)}$ for the FEA of each set of control parameters. For each output parameter a RS according to Eq. (7) is generated with the resulting torques and machine losses from the FEAs. Finally, the RSs for the total machine loss (Eq. (13)) and the torque error (Eq. (15)) outputs are both minimized with Eq. (10). The optimization can be performed by a particle swarm optimization (PSO) or a Pareto search algorithm. In order to investigate the error of the response surface and validate the optimal parameter sets, the two measures in Eq. (8) are applied.

\section{Simulation Results}

The investigated electrical machine is a two-phase SRG for 
Optimization of SPC based on DoE for SRMs (Duc Pham et al.)

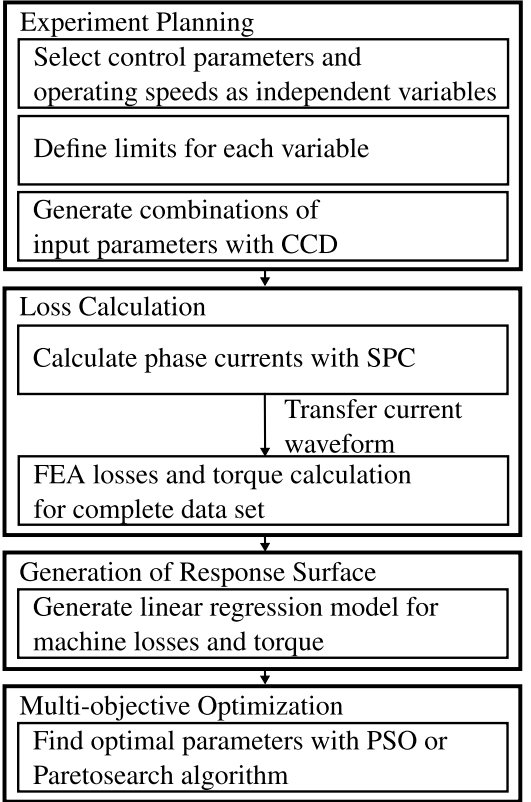

Fig. 4. Overview of applied workflow

Table 2. SRM Properties

\begin{tabular}{ll}
\hline Parameter & Value \\
\hline Diameter & $150 \mathrm{~mm}$ \\
Length & $200 \mathrm{~mm}$ \\
Number of windings & 40 \\
Wire diameter & $1.9 \mathrm{~mm}$ \\
Lamination thickness & $0.1 \mathrm{~mm}$ \\
Lamination resistivity & $52 \mu \Omega \mathrm{cm}$ \\
Dc-link voltage & $800 \mathrm{~V}$ \\
Nominal speed & $25 \mathrm{krpm}$ \\
Nominal power & $90 \mathrm{~kW}$ \\
\hline
\end{tabular}

a gas turbine application ${ }^{(11)}$. The electrical machine is mainly operated in generator mode. The active component of the machine consists of 8 stator teeth and 4 rotor teeth. Due to symmetry, a quarter of the machine is sufficient for the FEA as shown in Fig. 3. The asymmetrical rotor geometry allows a torque generation at standstill in order to start a gas turbine. The machine properties are shown in Table 2.

4.1 Goodness of Response Surfaces Two different design parameter sets are investigated. Each parameter set has a different number of design variables. The first set has four design parameters and also considers the machine speed $n_{\mathrm{SRG}}$. Thus, an arbitrary operating point $\left(n^{*} \mid T^{*}\right)$ can be considered in the MOO. The second set only consists of the three design parameters. The turn-off angle is set constant to $\theta_{\text {off }}=280^{\circ} \mathrm{el}$ for the complete loss calculation process. Generally, a different constant turn-off angle can be set. In this work, a constant value within the interval in Table 1 is selected. The two parameter sets mainly differ in the number of design parameters, which allows an investigation of the accuracy of the different RSs. While the first parameter set represents the maximum degree of freedom for selecting the optimal control parameters, the second one is a reduced and practical one, where either the turn-on or turn-off angle are assumed to be constant. Thus, the first set allows a greater solution space with more combinations of control parameters with minimum losses. However, more FEA calculations
Table 3. RMSE, $R^{2}$ and number of FEA runs for different parameter sets

\begin{tabular}{lcccc}
\hline Parameter Set & \multicolumn{3}{c}{ RMSE $\mid R^{2}$ in $\%$} & FEA Runs \\
& $T$ & $P_{\mathrm{Fe}}$ & $P_{\mathrm{Cu}}$ & $N_{\mathrm{CCD}}$ \\
\hline 1. $\theta_{\mathrm{on}}, \theta_{\mathrm{off}}, \Delta \theta_{\mathrm{fw}}, n_{\mathrm{SRG}}$ & $1.38 \mid 99.6$ & $3.33 \mid 99.9$ & $113 \mid 99.6$ & 25 \\
2. $\theta_{\mathrm{on}}, \Delta \theta_{\mathrm{fw}}, n_{\mathrm{SRG}}$ & $0.667 \mid 99.9$ & $3.76 \mid 99.9$ & $71.2 \mid 99.9$ & 15 \\
\hline
\end{tabular}

are necessary compared to the second set. An overview of the sets and the corresponding measures of error are shown in Table 3. The RMSE of the average torque between the RS and the FEA data set obtained by CCD decreases with less number of design variables. This indicates a lower prediction accuracy of the average torque for four design variables. However, the RMSE for the iron losses $P_{\mathrm{Fe}}$ increases, whereas the error for the copper losses $P_{\mathrm{Cu}}$ decreases for a lower number of design variables. Generally, the second-order RSs (see Eq. (7)) show high goodness for all sets. More than $99 \%$ of total variation can be described by the RSs. In the following, the set of RSs generated with four and three design variables are denoted as RS1 and RS2, respectively. Note that RSs representing windage losses are also generated. Due to their analytical calculation and their only dependency on the machine speed (see Eq. (5)), the $R^{2}$ values of the RSs are close to $100 \%$.

4.2 Validation of Optimized Design Parameters In this chapter, the precision of RS1 and RS2 are investigated. Thus, a validation operating area for the SRG is defined. The objective is to find control parameters for each operating point with minimized losses. Subsequently, additional FEAs validate the loss predictions from the RSs. One result of the MOO is depicted in Fig. 5. The optimal set of control parameters is located at the cross section of the Pareto front and the $x$-axis. Here, the difference of torque production and the reference torque is minimized (see Eq. (15)). Fig. 6 depicts the optimal control parameters for different reference speeds from $20 \mathrm{krpm}$ to $27.5 \mathrm{krpm}$ and powers from $18 \mathrm{~kW}$ to $90 \mathrm{~kW}$. The start of each bar denotes the turn-on angle $\theta_{\text {on }}$, whereas the end represents the turn-off angle $\theta_{\text {off }}$. The change of colors indicates the position of the freewheeling operation. Firstly, the RS1 including the machine speed is investigated (see on the left in Fig. 6). Intuitively, one would expect a smooth transition for all control angles in case of a constant operating speed and varying power outputs. However, the defined limits (see Table 1) cause discontinuities along varying power outputs. Another cause is the fact, that the control sets with three degrees of freedom for a specific torque output are not unique. In contrast, with RS2 the turn-on angle $\theta_{\text {on }}$ gradually increases with lower power generation for constant speed (see on the right in Fig. 6). In addition, the freewheeling duration $\Delta \theta_{\mathrm{fw}}$ slightly decreases for higher speeds.

Fig. 7 depicts the average torque output of the RS (blue), which is also the expected operating torque $T^{*}$, and the corresponding value obtained with FEA (orange). Note that the reference torque $T^{*}$ is given by the relation $P^{*}=2 \pi$. $n^{*} / 60 \cdot T^{*}$. Higher absolute torque errors $\Delta T$ occur for the reference power $P^{*}=90 \mathrm{~kW}$. The highest error occurs at $90 \mathrm{~kW}$ and $20 \mathrm{krpm}$ with an error of $3.75 \mathrm{Nm}$. Here, the RS overestimates the average machine torque. The lowest error in torque can be observed around the operating point 


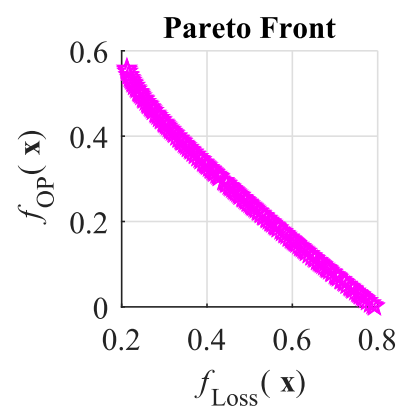

Fig. 5. Pareto front

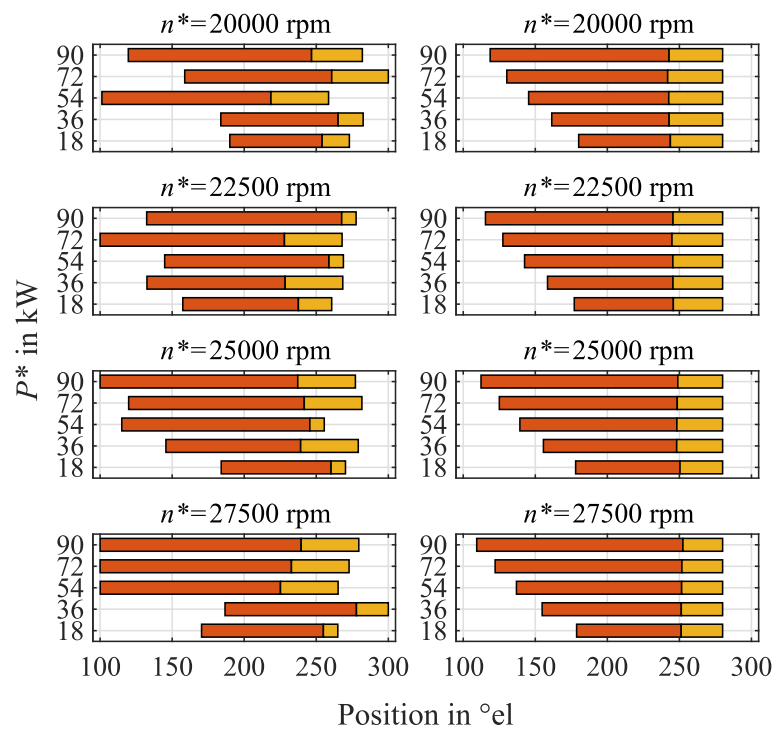

Fig. 6. Optimized SPC control parameters with RS1 on the left and RS2 on the right with const. $\theta_{\text {off }}=280^{\circ} \mathrm{el}$
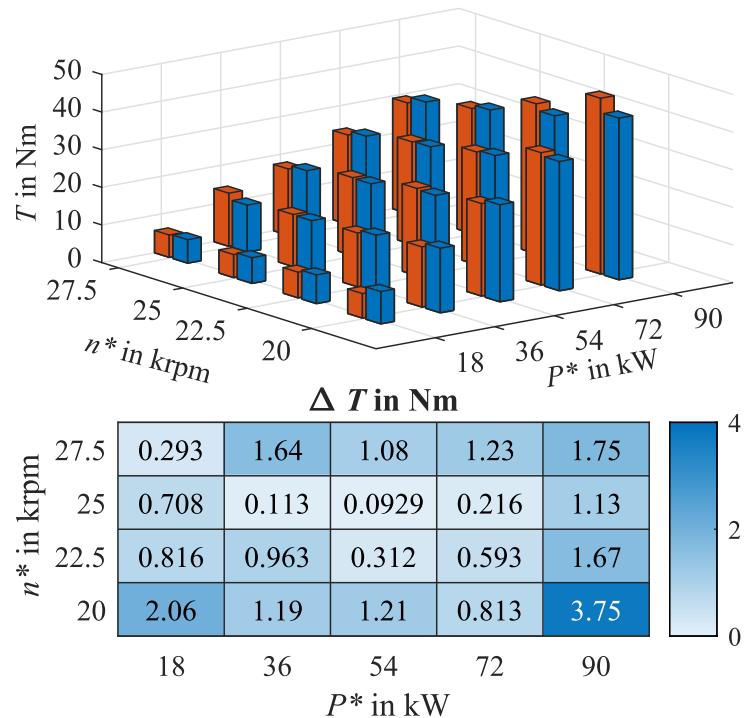

Fig. 7. Top: Average torque output $T_{\mathrm{m}, \mathrm{RS}}=T^{*}$ (blue) with RS1 and of torque $T_{\mathrm{m}, \text { Val }}$ (orange) from FEA, Bottom: Absolute error

$54 \mathrm{~kW}$ and $25 \mathrm{krpm}$. Fig. 8 shows the total machine losses approximated by the RSs (blue) and calculated by FEAs (orange). The maximum relative error of $6.47 \%$ is at the speed of $18 \mathrm{krpm}$. Here, higher deviations in the approximation of the iron and copper losses occur. The total losses in the nominal operating point is $P_{\text {Loss }}=3.7 \mathrm{~kW}$ and the error is

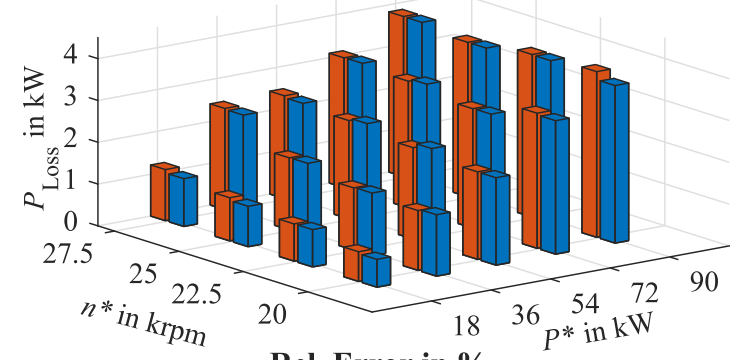

\begin{tabular}{|c|c|c|c|c|c|}
\hline \multirow{2}{*}{ ह $\begin{array}{r}27.5 \\
2\end{array}$} & \\
\hline & 6.47 & 0.848 & 1.77 & 0.24 & 0.0675 \\
\hline 吾 25 & 6.47 & 0.382 & 2.76 & 2.22 & 0.0423 \\
\hline $\begin{array}{l}\Xi 22.5 \\
* 2\end{array}$ & 0.303 & 1.02 & 5.34 & 0.25 & 0.197 \\
\hline 20 & 6.21 & 2.71 & 0.0741 & 1.26 & 4.89 \\
\hline & 18 & 36 & $\begin{array}{c}54 \\
P * \text { in } \mathrm{kV}\end{array}$ & 72 & 90 \\
\hline
\end{tabular}

Fig. 8. Top: Total machine loss output $P_{\mathrm{RS}}$ (blue) with RS1 and loss $P_{\text {Val }}$ (orange) from FEA, Bottom: Relative error

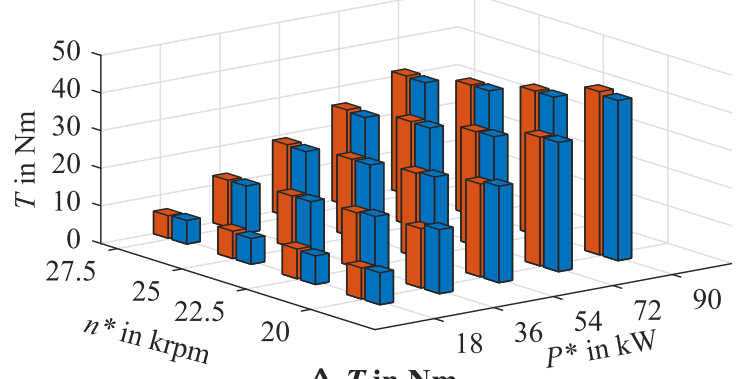

\begin{tabular}{|c|c|c|c|c|c|}
\hline \multirow{3}{*}{$\begin{array}{r}27.5 \\
25\end{array}$} & \multicolumn{5}{|c|}{$\Delta T$ in $N m$} \\
\hline & 0.243 & 0.0206 & 0.29 & 0.503 & 0.257 \\
\hline & 0.397 & 0.0503 & 0.007 & 0.161 & 0.0367 \\
\hline 22.5 & 0.277 & 0.538 & 0.536 & 0.212 & 0.0582 \\
\hline 20 & 0.272 & 1.26 & 0.933 & 0.14 & 0.8 \\
\hline & 18 & 36 & 54 & 72 & 90 \\
\hline
\end{tabular}

Fig. 9. Top: Average torque output $T_{\mathrm{m}, \mathrm{RS}}=T^{*}$ (blue) with RS2 and of torque $T_{\mathrm{m} \text {,Val }}$ (orange) from FEA, Bottom: Absolute error

$0.0423 \%$. However, due to the absolute error in torque of $\Delta T=1.13 \mathrm{Nm}$ the FEA for validation calculates a generating power of $P_{\mathrm{Val}}=87.01 \mathrm{~kW}$. Fig. 9 summarizes the torque output with RS2. Here, the turn-off angle $\theta_{\text {off }}$ is constant. Therefore, the RS is only a function of the machine speed $n_{\mathrm{SRG}}$, the turn-on angle $\theta_{\mathrm{on}}$ and the freewheeling length $\Delta \theta_{\mathrm{fw}}$. Due to the smaller solution space, the absolute torque error is generally reduced compared to Fig. 7. The highest error in torque of $1.26 \mathrm{Nm}$ occurs at a speed of $20 \mathrm{krpm}$ and at the desired generating power of $36 \mathrm{~kW}$. For the nominal operating point, the validation FEA calculates the total machine loss of $P_{\text {Val }}=3.82 \mathrm{~kW}$ (see Fig. 10). This is a relative error of $1.05 \%$ compared to the predicted total loss $P_{\mathrm{RS}}=3.86 \mathrm{~kW}$ obtained by $\mathrm{RS} 2$. The maximum error with three design variables is $6.3 \%$ for the operating point with $18 \mathrm{~kW}$ and $22.5 \mathrm{krpm}$. The mean error for all considered operating points is $1.9 \%$. In total, the mean error is smaller compared to RS1 (see Fig. 8) 


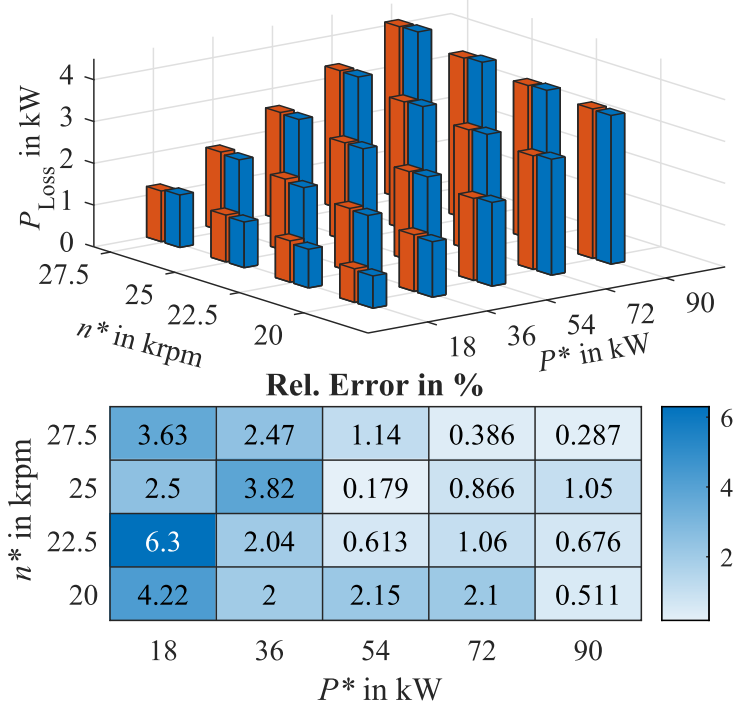

Fig. 10. Top: Total machine loss output $P_{\mathrm{RS}}$ (blue) with RS2 and loss $P_{\text {Val }}$ (orange) from FEA, Bottom: Relative error

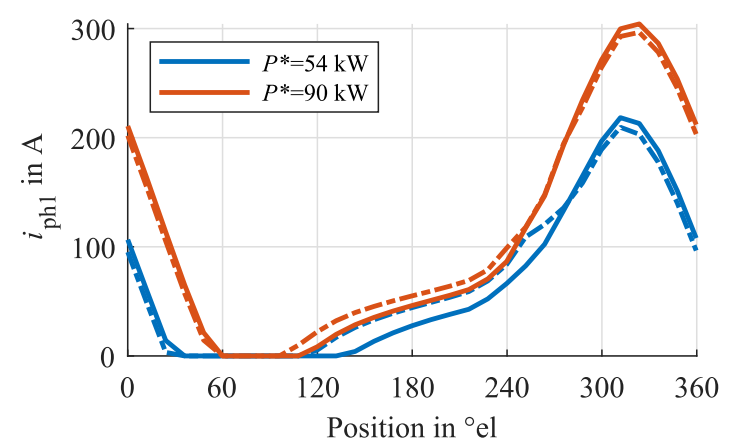

Fig. 11. Phase current with optimized parameter from RS1 (discontinuous) and from RS2 (continuous lines) at the power $P_{\mathrm{N}}=90 \mathrm{~kW}$ and $60 \% \cdot P_{\mathrm{N}}$ for speed $n_{\text {nom }}=25 \mathrm{krpm}$

with a mean error of $2.18 \%$. In Fig. 11, the current waveforms for the two reference generation power $54 \mathrm{~kW}$ and $90 \mathrm{~kW}$ at a constant speed of $n_{\text {nom }}=25 \mathrm{krpm}$ are shown. For each generation power, two current waveforms with different control angles are given. Fig. 6 provides the corresponding control parameters. For RS1 the turn-on angles and the freewheeling duration are smaller compared to RS2. As a result, the control based on RS1 leads to higher currents during the phase magnetization and lower currents as soon as freewheeling is active. The current waveforms show that the MOO results based on different RS lead to different optimal parameters. The error analyses show, that both RS1 and RS2 are able to predict the torque generation of an SRG and its total machine losses in a particular operating point. However, it also demonstrates the rather high torque deviations for RS 1 with four degrees of freedom. The interactions between all four design variables cannot be completely represented in the RS $T_{\mathrm{m}, \mathrm{RS}}$ for average torque prediction. The assumption of a constant turn-off angle $\theta_{\text {off }}$ leads to better torque predictions with RS2, which is also consistent with the RMSE in Table 3. Furthermore, the RMSE and $R^{2}$ indicates that with four variables a lower accuracy is achieved compared to three variables regarding the copper loss prediction. The difference in prediction accuracy is also observed for different operating points, because the copper losses significantly impact the total machine losses at high speed operation. Consequently, loss predictions with RS2 have a lower total error. In general, RSs statistically approximate the SRG regarding torque production and loss generation. However, if the data set generated by DOE covers the relevant operating points for a considered application, low errors in torque and a maximum error of $6.3 \%$ can be achieved.

Not only is the selection of the design variables important, but also the selection of their intervals for DOE. Within this work, a non- continuous decreasing turn-on angle for increasing machine power is observed for RS1 (see Fig. 6). Hence, for ensuring machine stability the reduced parameter set RS2 should be considered. Eventually, different approaches compared to CCD with more experiments can improve the data set. Consequently, higher order linear regression models can also improve the input and output relation.

\section{Conclusion}

This paper presents an algorithm based on design of experiments and multi-objective optimization in order to find optimized control parameters for high-speed switched reluctance machines. An inscribed central composite design generates a data set based on the turn-on angle, turn-off angle and the freewheeling duration for single pulse control of an SRG. Furthermore, consideration of the machine speed as another design variable allows a torque and loss prediction over a wide operating area around the nominal speed. Not only are the resulting control parameters able to reach the operating points with a maximum torque error of $1.26 \mathrm{Nm}$ for the reduced response surface, but they also keep the losses minimal with a mean error of $1.9 \%$ for the considered operating points.

\section{Acknowledgment}

The presented study has been carried out in the project HyFly (FKZ: 20Q1710B), funded by the German national research funding LuFo V (Luftfahrtforschungsprogramm). The authors would like to acknowledge the support of the German Ministry of Economics (BMWi).

\section{References}

( 1 ) B. Burkhart: "Switched reluctance generator for range extender applications: Design, control and evaluation", Dissertation, RWTH Aachen, Aachen (2018)

( 2 ) I. Kioskeridis and C. Mademlis: "Optimal efficiency control ofswitched reluctance generators", IEEE Transactions on Power Electronics, Vol.21, No.4, pp.1062-1071 (2006)

( 3 ) T. Kojima and R.W.D. Doncker: "Impact of zero-volt loop control on efficiency of switched reluctance motors", IEEE Transactions on Industry Applications, Vol.53, No.4, pp.3621-3634 (2017)

( 4 ) A.I. Khuri and S. Mukhopadhyay: "Response surface methodology", Wiley Interdisciplinary Reviews: Computational Statistics, Vol.2, No.2, pp.128149 (2010)

( 5 ) JSOL Corporation: "Jmag version 18.0 - user's manual iron loss formulas", Wiley Interdisciplinary Reviews: Computational Statistics, Tokyo (2019)

( 6 ) Cogent Power: "Hi-Lite NO10", [Online], available: https://www.tatasteele urope.com/ts/sites/default/files/tata-steel-hi-lite-electrical-steel-no10-1270ndatasheet-en.pdf (2020)

( 7 ) C. Carstensen: "Eddy currents in windings of switched reluctance machines", Dissertation, RWTH Aachen, Aachen (2007)

( 8 ) J.E. Vrancik: "Prediction of windage power loss in alternators", NASA Lewis Research Center, Cleveland, OH, United States, NASA Tech Brief, Apr. 1 
(1971)

( 9 ) The Math-Works Inc.: "MATLAB, 9.7.0.1216025 (R2019b)", Natick, Massachusetts: The Math-Works Inc. (2019)

(10) JSOL Corporation: "JMAG, Version 18.0.01zi", Chuo-ku, Tokyo, Japan: JSOL Corporation (2019)

(11) D. Pham, A. Klein-Hessling, and R.W.D. Doncker: "Control of a DC-DC converter as an active filter in combination with switched reluctance generators for gas turbine applications", AIAA Propulsion and Energy 2019 Forum, American Institute of Aeronautics and Astronautics (2019)

(12) D. Pham, L.V. Pugazhenthi, G.T. Götz, and R.W.D. Doncker: "Optimization of Single Pulse Control based on Design of Experiments for Switched Reluctance Machines", 2020 23rd International Conference on Electrical Machines and Systems (ICEMS) (2020)

Duc Pham (Non-member) received the B.Sc. and M.Sc. in Elec-

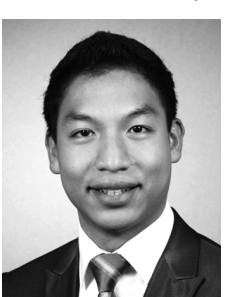
trical Engineering from RWTH Aachen University, Aachen, Germany, in 2015 and 2018, respectively. $\mathrm{He}$ is a research associate with the Institute for Power Electronics and Electrical Drives (ISEA) since 2018 $\mathrm{He}$ is working on electrical drives for electric and hybrid-electric propulsion systems focusing on thermal and loss analyses of switched reluctance machines and synchronous machines.

Leo Vijay Pugazhenthi (Non-member) received B.Eng. degree in

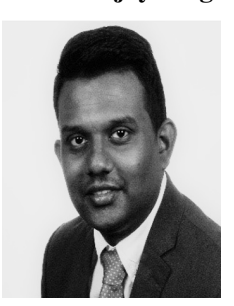
electrical and electronics engineering from Anna University, India in 2013 and M.Sc. in electrical power engineering from RWTH Aachen University, Germany in 2019. He is currently working as an Electrical Engineer in Valeo Siemens eAutomotive Germany GmbH, Bad Neustadt, Germany. His main activities are in the area of electromagnetic design of the electrical machines for eAutomotive. His research interests include electrical machine design and optimization algorithms at the system level.

Georg Tobias Götz (Non-member) received the B.Sc. and M.Sc. de-

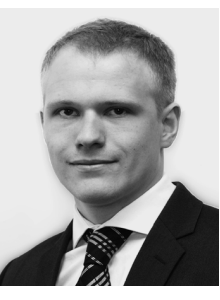
grees in electrical engineering from the Faculty of Electrical Engineering, Information Technology and Computer Engineering, RWTH Aachen University, Aachen, Germany, in 2016 and 2019, respectively. Since October 2019, he has been working as a research associate with the Institute for Power Electronics and Electrical Drives (ISEA), RWTH Aachen University, with a research focus on electrical drivetrains for mobility applications. He is member of the Research Training Group mobilEM, which researches in the field of Integrated Energy Supply Modules for Roadbound E-Mobility.
Rik W. De Doncker (Non-member) received the Ph.D. degree in elec-

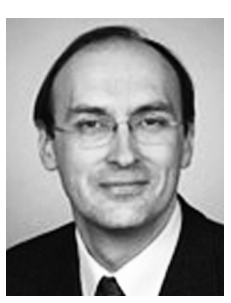
trical engineering from Katholieke Universiteit Leuven, Leuven, Belgium, in 1986. In 1987, he was appointed as a Visiting Associate Professor at the University of Wisconsin, Madison. After a short stay as an Adjunct Researcher with Interuniversity Microelectronics Centre, Leuven, he joined, in 1989, the Corporate Research and Development Center, General Electric Company, Schenectady, NY, USA. In 1994, he joined Silicon Power Corporation, a former division of General Electric, Inc., as the Vice President of Technology. In 1996, he became a Professor at RWTH Aachen University, Aachen, Germany, where he currently leads the Institute for Power Electronics and Electrical Drives (ISEA). Since 2006, he has been the Director of the E.ON Energy Research Center, RWTH Aachen University. He has authored more than 400 technical papers and is holder of more than 40 patents. Dr. De Doncker was the President of the IEEE Power Electronics Society (PELS) in 2005 and 2006. He was the founding Chairman of the German IEEE Industry Applications Society PELS Joint Chapter. In 2002, he was the recipient of the IEEE IAS Outstanding Achievement Award. In 2008, he received the IEEE PES Nari Hingorani Custom Power Award. In 2009, he led a VDE/ETG Task Force on Electric Vehicles. In 2010, he received an Honorary Doctor degree of TU Riga, Riga, Latvia. In 2013, he received the IEEE William E. Newell Power Electronics Award. In 2020, he received the IEEE Medal in Power Engineering. 